\title{
The developed consciousness model providing plausible guidelines to form a possible gross bridge between mind and matter, present physics and cognitive science, psychology and natural sciences, classical physics and quantum physics
}

\author{
Dhananjay Pal \\ Email address: \\ dhananjay.pal123@gmail.com,paldhananjay46@yahoo.com
}

Pharmacy College, Bengal School of Technology, Sugandha-Delhi Road, Chuchura, Dist.-Hooghly, West Bengal, INDIA, PIN-712 102

\section{To cite this article:}

Dhananjay Pal. The Developed Consciousness Model Providing Plausible Guidelines to Form a Possible Gross Bridge between Mind and Matter, Present Physics and Cognitive Science, Psychology and Natural Sciences, Classical Physics and Quantum Physics. American Journal of Physics and Applications. Vol. 1, No. 3, 2013, pp. 111-124. doi: 10.11648/j.ajpa.20130103.19

\begin{abstract}
A single field emerged at the origin of the universe, already containing within itself the blueprint of the physical universe. The primordial single field triggered the onset of the universe. Most physicists believe that a single super-force dominated the first instants of creation. Scientists have arrived at a simple but decisive conclusion that consciousness is very much a part of the universe, like other objects. Our consciousness model involving thought-carrying particle (TCP), thought retaining particle (TRP) and thought force (TF) signifies the existence of universal consciousness that exists along with the universe. This universal consciousness is a functional state of the universal mind (UM). This UM is evolved at the Big Bang from void. The UM is constituted by these TCP and TRP in the inherent presence of thought force (TF). Thought force (TF) is an expression of universal consciousness. The Thought force (TF) being the primordial quantum field functions as the original super-force. TF being the original super-force functions as the origin of all the fundamental fields. TCP is the carrier of thought force (TF) that, in turn, appears to be the origin of all the fields. The quantized energy $\left(\varepsilon_{T}\right)$ of TCP is responsible to cause the universal consciousness as well as the cosmic microwave background radiation temperature. The individual consciousness owes its origin to the universal consciousness created by the same $\varepsilon_{T}$. The same $\varepsilon_{T}$ is the energy responsible for generating thought force (TF). TF being an expression of the universal consciousness is applicable to any inanimate object as well as to any biological system (having thinking ability). The TF exerts its functions both in vitro and in vivo. Any matter as well as any individual mind is constituted by these TCP and TRP in the inherent presence of thought force (TF) in vitro and thought force (TF) in vivo and these TCP and TRP are originated from the same Void. These TCP, TRP and the thought force (TF) in vitro and thought force (TF) in vivo play significant roles in understanding matter, mind and consciousness. Theoretically observed coincidences through the consciousness model can provide plausible guidelines to form a possible gross bridge between mind and matter, present physics and cognitive science, psychology and natural sciences, classical physics and quantum physics.
\end{abstract}

Keywords: Void, Cosmic Microwave Background Radiation (CMBR), Universal Mind (UM), Thought Force ( $\mathrm{T}_{\mathrm{F}}$ ), Though-Carrying Particle (TCP), Thought Retaining Particle (TRP), Quantized Energy $\left(\boldsymbol{\varepsilon}_{T}\right)$ of TCP

\section{Introduction}

In Eastern philosophical traditions, consciousness is intrinsic to the universe, whereas in most Western views, consciousness is extrinsic, emerging from complex computation. How can these views be reconciled?

It is most relevant and important to indicate the names of various eminent physicists like Erwin Schrödinger, Eugene Wigner, Brian Josephson, John Wheeler, Roger Penrose, Henry P Stapp, Freeman J. Dyson, Paul Davies, David Bohm, Basil Hiley, Fritjof Capra, Fred Alan Wolf and Amit Goswami who have addressed the inclusion of consciousness in their work. Consciousness is to be taken into account.

In contrast to the usual linear sequence of matter, body, 
life, brain, mind, consciousness, here the proposed cyclic sequence is first universal consciousness (a functional state of the universal mind), and then matter, body, life, brain, and regeneration of mind and consciousness. The evolution of life with mind and consciousness is possible purely due to the inherent existence of universal consciousness which exists along with the universe. The human nervous system is evolved to provide an appropriate material structure to individualize the universal consciousness, a characteristic of reality, pervading all manifestations.

Consciousness model of Pal et al [1-3] involving TCP, TRP and thought force $\left(\mathrm{T}_{\mathrm{F}}\right)$ signifies the existence of universal consciousness that exists along with the universe. Pal et al [3] showed that this universal consciousness is a functional state of Universal Mind (UM). Pal et al [3] explained that the UM is evolved at the Big Bang from the eternal Void. This Void, in turn, is the source of infinite energy. And this UM is a finer matter. The individual mind being a constituent of the UM is also a finer matter. The constituents of the UM and individual mind are the same. The ultimate constituents of matter and mind are the same as both mind and matter are aspects of one fundamental reality, which is called UM. The brain is the mediating link or interface between the individual mind and body.

Pal et al [3] explained that the constituents of the UM are the ultimate constituents of matter itself as everything in this universe is a manifestation of this UM. Pal et al [3] expressed that the UM is constituted by these TCP and TRP in the inherent presence of thought force $\left(\mathrm{T}_{\mathrm{F}}\right)$. Pal et al $[1,3]$ further explained that the ultimate constituents of matter and mind are these TCP and TRP in the inherent presence of thought force $\left(\mathrm{T}_{\mathrm{F}}\right)$ in vitro and thought force $\left(\mathrm{T}_{\mathrm{F}}\right)$ in vivo.

Physicists determined that underlying quantum fields give birth to elementary particles. Bhaumik [4] mentioned that Frank Wilczek pointed out, "In quantum field theory, the primary elements of reality are not individual particles, but underlying fields. Thus, for example, all electrons are but excitations of an underlying field, naturally called electric field". The same holds true for all the fundamental particles of which matter is made.

The existence of matter depends on the existence of force and vice versa. TCP cannot exist without TRP and vice versa. Many physicists believe that unifying all the forces, including gravity, into a single theory would require a phenomenon called super-symmetry. With supersymmetry, every fermion would have a boson twin, and vice-versa. The thought force $\left(\mathrm{T}_{\mathrm{F}}\right)$ is carried by the TCP in the presence of its super-symmetrical partner TRP. TCP that behaves like boson should accompany its supersymmetrical partner TRP that functions like fermion in the generalized simpler way. It is to be noted that these TCP and TRP function like wavicle: wave-particle duality.

\section{Thought Force}

Pal et al [1,3] and Pal [5] explained the existence of thought force (TF). Thought force (TF), an expression of the universal consciousness, is the primordial quantum field that, in turn, functions as the primary unified field. This TF being an expression of the universal consciousness is applicable to any inanimate object as well as to any biological system (having thinking ability). Thus the TF being an expression of the universal consciousness exerts its functions both in vitro and in vivo.

Physicists determined that underlying quantum fields give birth to elementary particles. Pal [5] expressed that the thought force (TF) is the primordial quantum field. Thought force (TF) being the primordial quantum field functions as the primary unified field. Thought force (TF) being the primordial quantum field gives birth to TRP that appears to be the origin of all the matter particles. TCP is the carrier of thought force (TF) that, in turn, appears to be the origin of all the fields. TCP thus appears to be the origin of all the field particles.

In a purpose to involve both the non-living and living systems of the world, Pal [5] has shown the existences of these TCP, TRP and thought force (TF) in vitro and thought force (TF) in vivo. Anyone can call this TCP by any other name, but as the highly developed living system will have to be evolved in the universe in the long run and as the thought of highly developed living system appears to be a kind of force to be called the thought force (TF) in vivo, we considered it is wise to call it as TCP. Further, as the universe exists along with the universal consciousness that, in turn, is created by the quantized energy $\left(\varepsilon_{T}\right)$ of TCP, we had to use the term TCP.

Pal (5) expressed that the non-living system of the world is governed by the thought force (TF) in vitro and this Thought force (TF) in vitro gives rise to TF (micro), SNF, EMF, WNF, GF and TF (macro)

where $\mathrm{TF}($ micro $)=$ Thought force in microcosm, $\mathrm{SNF}=$ Strong nuclear force, $\mathrm{EMF}=$ Electromagnetic force, $\mathrm{WNF}=$ Weak nuclear force, $\mathrm{GF}=$ Gravitational force and $\mathrm{TF}$ (macro) $=$ Thought force in macrocosm. It is to be noted here that TF (micro) is a stronger force than the SNF and $\mathrm{TF}$ (macro) is a weaker force even than the GF.

Pal (5) also expressed that the living system of the world is governed by the thought force (TF) in vivo and this Thought force (TF) in vivo is a type of force that represents the biological 'thought' which is the action of mind. This 'thought' being a type of force controls the 'thought processes' involving the firing of neurons through the quantum mechanical activities of these TCP and TRP in the presence of consciousness. Consciousness in living organisms is a process which involves the quantum mechanical activities of these TCP and TRP, the ultimate constituents of any matter as well as any mind in the inherent presence of thought force (TF) in vitro and the thought force (TF) in vivo as indicated by Pal et al [3]. This consciousness, in turn, is the quantized energy $\left(\mathcal{E}_{T}\right)$ of TCP. The thought force (TF) in vivo is demonstrated in numerous experiments in which thought has an effect on a physical process (often known as mind over matter). This biological 'thought' is a type of force that can cause movement. Controlling movement through thought alone is observed in several experiments conducted by many scientists as indicated by Pal (5). These experiments thus 
signify the existence of thought force (TF) in vivo.

Pal [5] explained the existence of TF (micro) (= Thought force in microcosm). This TF (micro) is the strongest interaction (a new class of 'extra strong' interaction). It is stronger than SNF (Strong Nuclear Force).

Pal [5] has also shown the existence of TF (macro) (= Thought force in macrocosm). It is the "weakest force" which is much weaker even than the gravity.

$\mathrm{Pal}$ et al [3] developed three different equations expressing the quantized energy ( $\varepsilon_{T}$ ) of TCP. The value of $\varepsilon_{T}$ in one of the three equations is shown below:

$$
\varepsilon_{T}=
$$

$$
4.384 \times 10^{-16} \mathrm{erg} \equiv 2.73 \times 10^{-4} \mathrm{eV} \equiv 2.73 \mathrm{~K} \cong C M B R
$$

temperature $\equiv 2.725 \mathrm{~K}$.

This conversion of erg $\equiv \mathrm{eV} \equiv \mathrm{K}$ is given by Weisskopf [6] as follows:

$$
1 \mathrm{erg} \cong 0.6241807 \times 10^{12} \mathrm{eV} \text { and } 10^{-4} \mathrm{eV} \cong 1 \mathrm{~K}
$$

$\mathrm{Pal}$ et al [3] expressed that the CMBR temperature is due to $\varepsilon_{T}$, the quantized energy of TCP where

$$
\boldsymbol{\varepsilon}_{T}=
$$

$$
4.384 \times 10^{-16} \mathrm{erg} \equiv 2.73 \times 10^{-4} \mathrm{eV} \equiv 2.73 \mathrm{~K} \cong C M B R
$$
temperature $\equiv 2.725 \mathrm{~K}$.

As per Pal et al [3], it is interesting to note that all of the three different equations ultimately give rise to the same result that is equivalent to the CMBR temperature. A sort of relationship is thus observed between the $\varepsilon_{T}$ and the CMBR temperature. This coincidence is thus signifying a probable role of TCP on the maintenance of CMBR temperature. Further, this coincidence is also signifying the existence of these TCP in the presence of TRP. TCP cannot exist without TRP and vice versa. The presence of TCP, TRP and Thought force (TF) in the universe is thus indicated and expressed mathematically. At present we are unable to explain when and how these TCP and TRP decoupled from the primordial cosmic soup.

Pal et al $[1,3]$ showed that the quantized energy $\left(\varepsilon_{T}\right)$ of TCP is responsible to cause the universal consciousness as well as the cosmic microwave background radiation temperature. The individual consciousness owes its origin to the universal consciousness created by the same $\varepsilon_{T}$. Ultimately this $\varepsilon_{T}$ represents universal consciousness. The existence of CMBR temperature indicates the existence of the TCP in the presence of TRP. The existence of TCP ensures the existence of the thought force (TF). Further, this TF being an expression of the quantized energy $\left(\mathcal{E}_{T}\right)$ of TCP exerts its functions both in vitro and in vivo.

\section{What is Life?}

Life is a state of flux that is being maintained by a typical form of energy which is nothing but the consciousness itself. Pal et al [3] and Pal [7] showed that the quantized energy $\left(\varepsilon_{T}\right)$ of TCP is responsible to cause the universal consciousness as well as the cosmic microwave background radiation temperature. The individual consciousness owes its origin to the universal consciousness created by the same $\varepsilon_{T}$. Pal et al $[1,3]$ and Pal [7] explained that life may be defined as a state of functional manifestation of consciousness that, in turn, is the quantized energy ( $\varepsilon_{T}$ ) of TCP. This $\varepsilon_{T}$ represents universal consciousness.

Thus,

$$
\text { Life }=\mathrm{f}(\text { Consciousness })=\mathrm{f}\left(\boldsymbol{\varepsilon}_{T}\right)
$$

where $\boldsymbol{\varepsilon}_{T}=$ quantized energy of the TCP $=4.384 \times 10^{-16} \mathrm{erg}$.

$$
\varepsilon_{T}=h v_{T}=h c / \lambda_{T}=4.384 \times 10^{-16} \mathrm{erg}
$$

where $\varepsilon_{T}=$ quantized energy of the TCP $=4.384 \times 10^{-16} \mathrm{erg}$,

$$
\begin{aligned}
& \boldsymbol{v}_{T}=\text { frequency of the TCP } \\
& =\varepsilon_{T} / h=66.12 \times 10^{9} \mathrm{~Hz} .=66.12 \mathrm{GHz}, \\
& \mathrm{h}=\text { Planck's quantum constant }=6.63 \times 10^{-27} \mathrm{erg} . \mathrm{sec}, \\
& \mathrm{c}=\text { free-space velocity of light }=3 \times 10^{10} \mathrm{~cm} / \mathrm{sec}, \\
& \lambda_{T}=\text { wave-length of the } \mathrm{TCP}=0.4537 \mathrm{~cm} .
\end{aligned}
$$

\subsection{What is Consciousness?}

Psychologists, neuroscientists, philosophers, and other professionals continue to engage in an ongoing debate as to what consciousness means. In reality, we may never know. Is it a product of the biological and classical physical interactions of the human brain; or is it something more fundamental, perhaps electromagnetic, or the result of quantum physics principles that we don't yet fully understand? Could it be something even more profound than that -- something beyond the scope of science and physics, any kind of physics, for us to understand?

According to the ancient Vedanta, consciousness is not an emergent property of matter that comes into existence only through the functioning of the human nervous system. Instead, consciousness is a characteristic of reality, pervading all manifestations. This unbounded field of nature's universal consciousness is not limited to an individual consciousness. From this viewpoint, the role of the human nervous system is to provide an appropriate material structure to individualize the universal consciousness. It appears that inanimate matter itself cannot generate consciousness without the inherent existence of universal consciousness.

Pal et al [2] explained, "Consciousness is the realization of existence, and there are as many states of consciousness as there are states of existence. Every living being has a consciousness of its own depending on complexity of the brain and the activity of the viable numbers of TCP in the presence of TRP; and the state of its consciousness changes 
every moment of time. Consciousness is the perception of the relation it bears to things and as this relation changes, consciousness changes its character. Consciousness itself does not change; it only moves up and down on the 'scale of the realization' of existence through the 'sub-conscious', 'conscious' and 'super-conscious' states". There is another term called unconscious state. The most usual unconscious state is sleep. A deeper form of unconsciousness is called a coma.

\subsection{Consciousness May be Defined as the 'Self- Organized' Capability of Any Living Being to Activate TCP and TRP}

Pal et al [3] expressed that consciousness may be defined as the 'self-organized' capability of any living being to activate TCP and TRP, the ultimate constituents of mind and matter and to exert its functions. What can generate, maintain and activate TCP and TRP is called animate having 'active consciousness' and what cannot is called inanimate, i.e., devoid of active consciousness. As anything inanimate does neither have the power to activate the TCP and TRP nor have the capability to catalyze the activity of TCP and TRP, so it cannot generate consciousness. On the contrary, anything animate has the 'self-organized' power to generate, activate and catalyze the activity of TCP and TRP in order to generate and maintain consciousness along with the vital living force. Prigogine et al [8] explained "Self-Organization in Non-Equilibrium Systems".

Consciousness in living organisms is a process which involves the quantum mechanical activities of these TCP and TRP, the ultimate constituents of any matter as well as any mind in the inherent presence of thought force $\left(\mathrm{T}_{\mathrm{F}}\right)$ in vitro and the thought force $\left(\mathrm{T}_{\mathrm{F}}\right)$ in vivo as indicated by $\mathrm{Pal}$ et al $[1,3]$. And these TCP and TRP govern the activities of neurons (not the other way round). Neurons are simply the equipments used to generate consciousness and awareness. The consciousness itself is functioning as an inter-linking agent between the animate and inanimate through the quantum mechanical activities of these TCP and TRP indicated by [3].

\subsection{Existence of Universal Consciousness}

Pal et al [3] and Pal [7] showed the existence of universal consciousness and explained that the quantized energy $\left(\varepsilon_{T}\right)$ of TCP is responsible to cause the universal consciousness as well as the cosmic microwave background radiation temperature. The individual consciousness owes its origin to the universal consciousness created by the same $\varepsilon_{T}$. Many physicists agree with the idea that consciousness is non-local, fundamental in the universe and consciousness is very much a part of the universe, like other objects. It is to be noted that $\mathcal{E}_{T}$, the quantized energy of TCP represents universal consciousness.

Nelson [9], Director of Global Consciousness Project, has observed through the experimentation that coherent consciousness creates order in the world; and subtle interactions link us with each other and the Earth. When human consciousness becomes coherent and synchronized, the behavior of random systems may change. Quantum event based random number generators (RNGs) produce completely unpredictable sequences of zeroes and ones. But when a great event synchronizes the feelings of millions of people, our network of RNGs becomes subtly structured. The probability is less than one in a billion that the effect is due to chance. The evidence suggests an emerging noosphere, or the unifying field of consciousness described by sages in all cultures. This evidence signifies and proves the existence of universal consciousness.

The existence of universal consciousness is explained by Radin [10] through experimentation as it is expressed in his book The Conscious Universe: The Scientific Truth of Psychic Phenomena. Further, Consciousness, not matter, is the ground of all existence, declares University of Oregon physicist Goswami through his published (1993) book, "The Self-Aware Universe: How Consciousness Creates the Material World".

As per Penrose [11], consciousness is a part of the universe. Van De Bogart [12] explained, "Since consciousness is a part of the universe it then follows that all consciousness, and the universe, are of the same matrix of energy fields".

\section{Possible Relation of Consciousness with Mind}

Modern scientists have not even been able to arrive at a consensus on what should be a definition of the totality of consciousness. The brain is intricately linked to the process of consciousness and consciousness is thought to be a phenomenon of the mind.

\section{It appears that}

$$
\text { Consciousness }=\mathrm{f}(\text { mind })
$$

Consciousness is the functional state of mind. Presence of consciousness signifies the presence of mind and vice versa.

\section{It is apparent that}

$$
\text { Animate } \neq \text { Inanimate }
$$

Three critical factors which distinguish life from nonliving are consciousness, metabolism and reproduction. (4):

In a gross presentation, we can express from the equation

$$
\text { Animate }- \text { Consciousness }=\text { Inanimate }
$$

Scientists would have to define and characterize consciousness properly. We should have the proper knowledge about the exact characteristics of consciousness in order to address many present day scientific enigmas. 


\section{What is the Exact Definition of Mind? What is Mind?}

We observe a great controversy concerning the exact definition of mind. There are two apparently opposite views: one is the substantial view and the other is the functional view.

In the substantial view, the mind is a type of substance. Mind is a finer matter having an autonomous existence. Mind is a single entity, perhaps having its base in the brain but distinct from it. In its most extreme form as in the Indian Upanishads and Vedanta, the mind is not only a finer matter but also it is an entity wholly separate from the body, in fact a manifestation of the soul, which will survive the body's death in the form of the spiritual body or mental body [called Linga Sharira or Sukshma Sharira in Sanskrit] as expressed by Vivekananda [13] who indicated that this mental body bears all the mental impressions. This mental body is also called 'spirit'.

In the functional view, the mind is closely related to the functions of the brain and can have no autonomous existence beyond the brain, nor can they survive its death. In this view, mind is a 'state' created by the activities of the brain with other parts of the nervous system as it is indicated by cognitive neuro-scientists and Artificial Intelligence (AI) scientists. According to them 'minds are simply what brains do'. In this functional view, mind is a subjective manifestation of consciousness: the human brain's ability to be aware of its own existence. The concept of the mind is therefore a means by which the conscious brain understands its own operations.

The modern cognitive neuroscientists generally accept the fact that the "mind" is not an isolated entity and the mind is a state created by the activities of brain with other parts of the nervous system (CNS, PNS along with ANS). Although the mind is generally accepted to be an abstract having no spatial location or public observability, yet it is a type of fine matter according to the ancient Indian Upanishads and Vedanta. The Vedanta indicates that the universe exists along with the universal consciousness. Pal et al $[1,3]$ explained that this universal consciousness is a functional state of the Universal Mind (UM). This UM is constituted by these TCP and TRP which, in turn, are the ultimate constituents of any matter and any mind in the inherent presence of thought force (TF) in vitro and thought force (TF) in vivo. It is to be noted that these TCP and TRP are the ultimate constituents of any matter in the inherent presence of thought force (TF) in vitro. And these TCP and TRP are the ultimate constituents of any mind in the inherent presence of thought force (TF) in vivo. Pal et al [1, 3] explained that any matter as well as any individual mind is constituted by these TCP and TRP in the inherent presence of Thought force (TF) in vitro and Thought force (TF) in vivo.

Modern Scientists are not yet able to understand how the brain works to make the mind. They know that brain has got neurons that communicate across synapses by releasing a neurotransmitter, and that generates electrical impulses, and the receiving neuron then talks to its neighbor neurons the same way. If the mind depends on the brain, then all aspects of the mind are going to depend on these simple electrical, chemical processes. According to this concept, the existence of the UM requires the existence of the universal brain to form the UM. The concept of universal brain is not logically acceptable. Thus the concept that the mind depends on the brain becomes questionable if the existence of the UM is valid.

It appears that scientists would have to decide in a purpose to conclude clearly whether mind is a 'state' (created by the activities of the brain with other parts of the nervous system as it is indicated by the functional view of mind) or 'a finer matter' (as it is indicated by the Indian Upanishads and Vedanta).

\section{Special Characteristics of Mind and Consciousness}

1. The basic mystery of the mind is how does it emerge from pure matter? How do those units that are made of tiny particles, give rise to the unique and essentially private, experience called consciousness? Do the particles that constitute our brain determine what we think and do? Do these tiny particles ultimately govern the thinking ability of the bioelectrical system? Or, are we free to have our own will? Is consciousness just froth sitting on top of the brain's electronics? How consciousness is being operated? Why should a bunch of atoms have thinking ability? Gross answers to all these inquiries are possible if we accept these TCP and TRP as the ultimate constituents of mind and matter in the inherent presence of thought force $\left(\mathrm{T}_{\mathrm{F}}\right)$ in vivo and thought force $\left(\mathrm{T}_{\mathrm{F}}\right)$ in vitro as indicated by Pal et al [3]. It is to be noted that the thought force $\left(\mathrm{T}_{\mathrm{F}}\right)$ being a primordial quantum field exerts its functions both in vitro and in vivo.

2. Modern Scientists are not yet able to understand how the brain works to make the mind. They know that brain has got neurons that communicate across synapses by releasing a neurotransmitter, and that generates electrical impulses, and the receiving neuron then talks to its neighbors the same way. If the mind depends on the brain, then all aspects of the mind are going to depend on these simple electrical, chemical processes. According to this concept, the existence of the UM requires the existence of the universal brain to form the UM. The concept of universal brain is not logically acceptable. Thus the concept that the mind depends on the brain becomes questionable if the existence of the UM is valid.

3. We are what our minds make us. The mind is a very powerful controller of the body. The mind controls everything as indicated by Pal et al [1, 3] and Pal [7]. Mind is conditioned from the time we are young. It gives our abilities, our perceptions, our character and the way we think. From science to religion, from politics to economics, everything is controlled by the human mind. We perceive, we believe and we react. Things that matter are what we think they are. The 
quest for truth is bafflingly elusive, after all what is the 'truth'? We have come to the realization that if I believe it is true, it indeed is true for me, regardless of how incredible you may think it is. Experiments do not matter, arguments do not matter, reality does not matter, in fact, matter does not matter---it is all in the mind. As a joker succinctly put it, "It is a case of mind over matter-I do not mind and you do not matter".

4. Consciousness model of Pal et al [1-3] involving TCP, TRP and thought force $\left(\mathrm{T}_{\mathrm{F}}\right)$ signifies the existence of universal consciousness that exists along with the universe. Pal et al [3] showed that this universal consciousness is a functional state of Universal Mind (UM). Pal et al [3] explained that the UM is evolved at the Big Bang from the eternal Void. This Void, in turn, is the source of infinite energy. And this UM is a finer matter. The individual mind being a constituent of the UM is also a finer matter. The constituents of the UM and individual mind are the same. The ultimate constituents of matter and mind are the same as both mind and matter are aspects of one fundamental reality, which is called UM. The brain is the mediating link or interface between the individual mind and body. Pal et al [3] explained that the constituents of the UM are the ultimate constituents of matter itself as everything in this universe is a manifestation of this UM. Pal et al [3] expressed that the UM is constituted by these TCP and TRP in the inherent presence of thought force $\left(\mathrm{T}_{\mathrm{F}}\right)$. Pal et al $[1,3]$ further explained that the ultimate constituents of matter and mind are these TCP and TRP in the inherent presence of thought force $\left(\mathrm{T}_{\mathrm{F}}\right)$ in vitro and thought force $\left(\mathrm{T}_{\mathrm{F}}\right)$ in vivo.

5. Current scientific views regarding the origin of consciousness vary widely and range from an 'epiphenomenon' arising from neuronal networks, to neuronal quantum processes, to a separate undiscovered scientific entity.

6. Consciousness is the functional state of mind. Presence of consciousness signifies the presence of mind and vice versa. It is to be understood that consciousness is the realization of existence and consciousness moves up and down on the 'scale of the realization' of existence through the 'sub-conscious', 'conscious' and 'super-conscious' states as indicated by Pal et al [2].

7. Consciousness may be defined as the 'self-organized' capability of any living being to activate TCP and TRP, the ultimate constituents of mind and matter and to exert its functions. What can generate, maintain and activate TCP and TRP is called animate having 'active consciousness' and what cannot is called inanimate, i.e., devoid of active consciousness.

8. Continuity of consciousness is found through the experimental observations of Parnia[14], Lommel [15], Fenwick [16] and Beauregard \& O'Leary [17 \& 18] and demonstrate the existence of consciousness during the cessation of brain activity and thus support the existence of universal consciousness as well as substantial view of mind as indicated by the Indian Upanishads and Vedanta.

9. Everything in this universe is interlinked and intertwined through the existence of the quantum mechanical activities of these TCP in the inherent presence of TRP. Pal et al [1,3] showed that the quantized energy $\left(\varepsilon_{T}\right)$ of TCP is responsible to cause the universal consciousness as well as the cosmic microwave background radiation temperature. The individual consciousness owes its origin to the universal consciousness created by the same $\varepsilon_{T}$.

Ultimately this $\mathcal{E}_{T}$ represents universal consciousness. This universal consciousness functions as a universe wide web (uww) covering the universe as a whole with all its parameters (including void) and inhabitants (with or without consciousness). In this picture, uww of consciousness, quantum concepts like wave particle dualism, position momentum uncertainty, non-locality and concept of unified field become somewhat understandable as all the entities of this universe are interlinked and intertwined. In this picture, the universal consciousness replaces ether of yesteryears and contains the whole of the universe in its fold. The 'spooky action-at-a-distance' may thus be built in nature through the existence of these TCP and TRP in the presence of thought force $\left(\mathrm{T}_{\mathrm{F}}\right)$.

10. In a purpose to conclude clearly, it appears, that scientists would have to decide whether mind is a 'state' (created by the activities of the brain with other parts of the nervous system as it is indicated by the functional view of mind) or 'a finer matter' (as it is indicated by the Indian Upanishads and Vedanta).

\section{Plausible Drawbacks of the Functional View of Mind}

1. The science of the mind may be connected with the science of the body. It is possible that the modern mind has been developed to get its present shape by the directive of the UM. The mind is an all-purpose computer responding impartially to the world around it; and this mind had, through natural selection, evolved to respond in a particular way to particular environmental circumstances. Humans are 'adaptation executors' carrying out programs written into the mind's machinery long ago. "The machinery does not know its own programming", said Cosmides and Tooby [19-21], intellectual leading evolutionary psychologists. The evolutionary psychologists see the mind as preprogrammed, made up of specialized mechanisms--'modules' or 'organs'. The evolutionary psychologists argue that their job is to approach the mind as an ancient engineering project, developing and testing out hypotheses about what 'designed problems' needed solving. Evolutionary psychologists thus signify the existence of the UM. 
Such an approach offers a badly needed bridge between psychology and the natural sciences. A plausible bridge between psychology and the natural sciences can be achieved through the developed consciousness model.

2. Growing evidence of the continuity of consciousness demonstrates the existence of consciousness during the cessation of brain activity. In a purpose to determine the nature of human mind and consciousness as well as its relationship with the brain, Parnia[14], Lommel [15], Fenwick [16] and Beauregard \& O'Leary [17 \& 18] observed through different lines of experiments and through studies of cardiac arrest survivors that the cerebral functioning as measured by electrical activity of the brain ceases during cardiac arrest. Sam Parnia [14] observed in the cardiac arrest survivors that the human mind and consciousness continue to function in the absence of brain function when there is cardiac arrest. Thus observations in these studies of cardiac arrest survivors raise the possibility that human mind and consciousness may continue to function in the absence of brain function. This indicates the existence of universal consciousness. The nervous system is evolved to individualize this universal consciousness that, in turn, exists along with the universe. These experimental observations support the substantial view of mind as indicated by the Indian Upanishads and Vedanta.

3. Continuity of consciousness indicates the existence of universal consciousness that exists along with the universe. This universal consciousness is to be taken into account, but usually ignored. Many physicists agree with the idea that consciousness is non-local, fundamental in the universe and consciousness is very much a part of the universe, like other objects. This universal consciousness is a functional state of the UM that, in turn, is a finer matter. Both, mind and matter are aspects of one fundamental and underlying reality, which is called Universal Mind (UM). The functional state of the $\mathrm{UM}$ is the universal consciousness that exists along with the universe. It appears that inanimate matter itself cannot generate consciousness without the inherent existence of this universal consciousness.

4. As per the ancient Vedanta, everything in this universe is a manifestation of the UM. This UM is a finer matter. The individual mind being a constituent of the UM is also a finer matter. The constituents of the UM and individual mind are the same. The ultimate constituents of matter and mind are the same as both mind and matter are aspects of one fundamental reality, which is called UM. The brain is the mediating link or interface between the individual mind and body.

5. Pal et al [3] explained that the constituents of the $\mathrm{UM}$ are the ultimate constituents of matter itself as everything in this universe is a manifestation of this UM. Pal et al [3] expressed that the UM is constituted by these TCP and TRP in the inherent presence of thought force $\left(\mathrm{T}_{\mathrm{F}}\right)$. Pal et al $[1,3]$ further explained that the ultimate constituents of matter and mind are these TCP and TRP in the inherent presence of thought force $\left(\mathrm{T}_{\mathrm{F}}\right)$ in vitro and thought force $\left(\mathrm{T}_{\mathrm{F}}\right)$ in vivo.

6. According to the Vedanta, consciousness is not an emergent property of matter that comes into existence only through the functioning of the human nervous system. Instead, consciousness is a characteristic of reality, pervading all manifestations. This unbounded field of nature's universal consciousness is not limited to an individual consciousness. From this viewpoint, the role of the human nervous system is to provide an appropriate material structure to individualize this universal consciousness.

7. The human nervous system is evolved to provide an appropriate material structure to individualize the universal consciousness, which is a characteristic of reality, pervading all manifestations. This unbounded field of nature's 'universal consciousness' is not limited to an individual consciousness.

8. We are what our minds make us. The mind is a very powerful controller of the body. It appears that the mind controls everything. Mind exerts its role from the very beginning of life. It governs our abilities, our faculties, our perceptions, our attitudes, our character and the way we become habituated to think through the utilization of the instrumental brain.

9. Freeman J. Dyson said, "It appears that mind, as manifested by the capacity to make choices, is to some extent inherent in every atom".

10. If mind has the capacity to make choices then this quality is demonstrated by atoms which make unpredictable choices between alternate possibilities according to the laws of quantum mechanics. "If the quality of mind is present at the atomic level, this suggests a return to the Vedantic concept of a Universal Mind (UM) which pervades all matter: a concept in agreement with the conclusion of University of Oregon physicist Amit Goswami. This UM must have been present at the inception of the universe".

11. Consciousness, not matter, is the ground of all existence, declares University of Oregon physicist Goswami, echoing the mystic sages of his native India. He holds that the universe is self-aware, and that consciousness creates the physical world. Matter is an expression of mind, not separate from mind, but mind manifested materially.

12. Example of three friends with different mental qualities. Let us take the popular example of a case of three friends visiting an ocean. If it is observed that one of them is frightened, other one is delighted and third person remains indifferent on the first sight of an ocean provided that they have 
not come in contact with the ocean before, then what should be the interpretation of mind that is related to the functions of the brain? These three different persons use their eyes and brains as the same type of instrument to look at the ocean. Here, the usual functioning of brain mechanism is not producing the same result on these three specified persons. The differences in the qualitative experiences are not due to the different functioning of the brain mechanism. The qualitative feelings are absolutely personal and mental. The brain is functioning as an instrument to generate different types of qualitative feelings in the different persons depending on their mental status. This mental status of a man is the result of the sum total of all the previous mental impressions (of previous lives) that he is not able to remember in this present life, because he has already lost all the previous brains. Further, this mental status is not the outcome of the activities of the brain with the other parts of the nervous system. The mind or the mental status does not depend on the activity of the brain. All our qualities are basically mental. If the brain is the cause and the mind is the effect, then who is controlling our mental qualities? The brain does not generate and control mental qualities. The brain is simply the biologically evolved fine instrument through which the mental impressions as well as mental qualities are expressed and maintained.

13. We sometimes use the terms like 'beautiful mind' or 'vicious mind'. When we say that John Nash has a 'beautiful mind' (vide the cinema of same name), we do not simply mean that he has a beautiful brain only. On the contrary, we like to indicate that he has a special power within himself to utilize his brain for producing beautiful results for which we conclude that he has a 'beautiful mind'. In other words, the mental status or the inner mental power of John Nash enforces him to apply his brain in such a special way that the ultimate result is beautiful. The brain is nothing but a fine instrument through which the mind exerts its functions and the mind possesses the inner power (or mental qualities). When the mind desires to do something as per its mental impressions, it exerts its functions through the brain with other parts of the nervous system.

14. Driving force of love and hate. We always find the action of 'driving forces' like "Attractive and Repulsive forces" which are the outcome of the emotional power of love, hate, fear and other emotional aspects of the mental world. Mind and consciousness generate and control all these driving forces. I would not like to go to a person whom I hate but I would be eager to go to a person whom I personally love. This emotional aspect of my mental world is controlling my physical movements through my nervous system. These vital interactions manifest their actions in the external physical world through the movements of the physical body. And these active interactions are controlling the physical movements of almost all the living beings, thus signifying the fact that the mind is a very powerful controller of the body.

15. Mind and mental qualities control the body through the utilization of the brain with other parts of the nervous system in the presence of sense organs. Strokes and fatal accidents sometimes cause complete paralysis but leave 'thought processes' of the victims untouched, trapping the 'active mind' in a motionless body. It indicates that the brain is functioning as the inter-linking device between the body and mind. It is also to be noted that human beings in 'coma' state do not exhibit significant consciousness in the presence of 'life function'. This is due to the malfunction of the brain that is the proper instrument through which the mind exerts its functions with the help of TCP and TRP. These TCP and TRP are ultimately responsible to generate consciousness and awareness. Pal et al [3] expressed that the quantized energy ( $\varepsilon_{T}$ ) of the TCP represents consciousness.

16. Soul (Void) exerts its functions through the finer instrument called mind. This mind, in turn, exerts its functions through the brain with other parts of nervous system (CNS, PNS along with ANS).

17. The eternal Void has been covered by the mind to function as the Soul of the individual being. In the

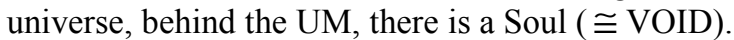
In the individual, behind the individual mind (which is a constituent of the UM), there is also a Soul ( $\cong$ VOID). Any matter as well as any individual mind is constituted by these TCP and TRP that, in turn, are originated from the same eternal Void. Thus the individual mind is operated by these TCP and TRP that, in turn, are ultimately governed and operated by the same eternal Void. The individual mind being a constituent of the UM has covered the eternal Void to serve the role of individual 'Soul'. This eternal Void is thus the common source and the source is still with us. This Void serves the role of Soul being covered by the mind. The mind-brain and brain-body links which are addressed by psychology, cognitive science, neuroscience and neurophysiology are, in our proposition, ultimately governed by the quantum mechanical activities of these TCP and TRP in the inherent presence of thought force $\left(\mathrm{T}_{\mathrm{F}}\right)$ in vitro and thought force $\left(\mathrm{T}_{\mathrm{F}}\right)$ in vivo. Consciousness that signifies the realization of existence is a functional state of mind.

18. This Soul ( $\cong$ VOID) being the master ruler of the body exerts its functions through the finer instrument called mind. This mind (with the help of TCP and TRP), in turn, exerts its functions through the brain with other parts of nervous system (CNS, PNS along with ANS) in the biological systems in order to grasp this physical universe, of course, in 
the inherent presence of consciousness. This consciousness is created and maintained by the quantum mechanical activities of these TCP in the inherent presence of TRP. Thus any matter (as well as mind) being ultimately composed of these TCP and TRP should have some sort of 'mind' as Dyson indicated. The more the development of the organized brain, the more the degree of manifestation of consciousness indicated by $\mathrm{Pal}$ et al [2]. This consciousness itself is acting as an interlinking agent between the animate and inanimate through the quantum mechanical activities of these TCP in the presence of TRP. Thus these TCP and TRP being the ultimate constituents of mind as well as matter play the most significant roles in many present day scientific enigmas.

19. Consciousness in living organisms is a process which involves the quantum mechanical activities of these TCP and TRP, the ultimate constituents of matter and mind in the inherent presence of thought force $\left(\mathrm{T}_{\mathrm{F}}\right)$ in vitro and thought force $\left(\mathrm{T}_{\mathrm{F}}\right)$ in vivo indicated by Pal et al [1-3]. And these TCP and TRP govern the activities of neurons (not the other way round). These neurons are simply the equipments used to generate consciousness and awareness. The consciousness itself is functioning as an interlinking agent between the animate and inanimate.

20. The quantum mechanical activities of these TCP and TRP could also explain self-organization.

21. Josephson et al [22] explained that both psychokinesis and telepathy might be found in quantum physics. It is our view that both psychokinesis and telepathy can be thoroughly interpreted through the consciousness model involving the quantum mechanical activities of TCP and TRP in the inherent presence of thought force $\left(\mathrm{T}_{\mathrm{F}}\right)$ in vitro and the thought force $\left(\mathrm{T}_{\mathrm{F}}\right)$ in vivo.

22. It appears that the development of 'selforganization' is possible through the generation and maintenance of consciousness by the quantum mechanical activities of these TCP and TRP, the ultimate constituents of matter and mind in the inherent presence of thought force $\left(\mathrm{T}_{\mathrm{F}}\right)$ in vitro and thought force $\left(\mathrm{T}_{\mathrm{F}}\right)$ in vivo.

23. Conclusion of Parnia [14] is human consciousness may work independently of the brain. Just because the brain is damaged does not necessarily mean that the "mind" is affected. This observation indicates that consciousness owes its origin to the universal consciousness. This consciousness, in turn, exists along with the universe; and thus consciousness is independent of the brain. Damaged brain does not necessarily mean that the "mind" is affected, thereby, signifies the substantial view of mind but not the functional view of mind.

24. The notions of a person's "spirit" and "soul" often overlap, as both contrast with body and both are imagined as surviving the bodily death in religion and occultism, and "spirit" can also have the sense of "ghost" (such as demons or deities) i.e. manifestations of the spirit of a deceased person. Barking of a pet dog looking at the window in a purpose to threaten and drive out 'something unwanted' appears to indicate the probable presence of something or someone that is nothing but a 'spirit' which is a spiritual body or mental body [called Linga Sharira or Sukshma Sharira in Sanskrit] indicated by Vivekananda [13]. Usually the human eye cannot recognize this spirit or mental body, but dogs and cats can do so. Functional view of mind cannot explain the possible existence of a spirit or a mental body.

\section{Formation of a Possible Gross Bridge between Mind and Matter, Present Physics and Cognitive Science, Psychology and Natural Sciences, Classical Physics and Quantum Physics through the Consciousness Model}

In a purpose to form a possible bridge between mind and matter, Pal [23] published a paper entitled, "Possible bridge between the mind and matter: The physical world is nothing but a projection of the mind consisting of TCP and TRP that, in turn, are the ultimate constituents of any matter also"

Pal [24] published a paper entitled, "Interpretation of quantum measurement as well as cognitive problem through the consciousness model" for showing a possible bridge between the present physics and cognitive science.

Pal [25] published a paper entitled, "Interpretation of the paranormal power like Extra Sensory Perception (ESP) and Psycho-kinesis (PK) through the consciousness model" for showing a possible bridge between the psychology and natural sciences.

Pal [26] published a paper entitled, "Possible bridging the classical physics and quantum physics through consciousness" for showing the formation of a possible bridge between the classical physics and quantum physics.

In a purpose to show a possible relation of cosmological constant $(\Lambda)$ with the TF (macro) [thought force in macrocosm] for the expansion of the universe, Pal [27] published a paper entitled, "Interpretation of the cause for the expansion of universe having its correlation with the cosmological constant $(\Lambda)$ through the consciousness model".

\subsection{Thought Force}

Pal et al [1,3] and Pal [5] explained that the quantized energy $\left(\varepsilon_{T}\right)$ of TCP is the energy responsible for generating thought force $\left(\mathrm{T}_{\mathrm{F}}\right)$; thus the $\mathrm{T}_{\mathrm{F}}$ may be expressed as 


$$
\mathrm{T}_{\mathrm{F}}=\varepsilon_{T} / \mathrm{D}_{\mathrm{i}}
$$

where $\mathrm{Di}=$ Interacting distance.

The thought force (TF) has been postulated to be carried by TCP in the inherent presence of TRP. The TCP being the carrier of the TF would behave like bosons when TRP would function like fermions.

Depending on the Di (= Interacting distance), it is possible to calculate and identify two new forces viz. TF (micro) [ $=$ Thought force in microcosm] and TF (macro) [= Thought force in macrocosm], the existence of which is indicated and expressed by Pal [5].

\subsubsection{Application of the Consciousness Model Involving TCP, TRP and $T_{F}$ (macro) to Explain the Cause for the Expansion of Universe [Having Its Correlation with the Cosmological Constant $(\Lambda)]$}

Pal [27] expressed: Einstein gave us his cosmological constant, which he considered a repulsive force equal but opposite to gravity. The majority of scientists today believe this cosmological constant repulsive force does indeed exist between all the stars and galaxies holding them apart.

The cosmological constant is estimated by cosmologists to be on the order of $10^{-29} \mathrm{~g} / \mathrm{cm}^{3}$, or about $10^{-120}$ in reduced Planck units.

It is to be noted that that both the TCP and TRP are interchangeable at 'super-symmetry' as they have the same quantized energy and as they behave like biophotons in animals and like simple photons in inanimate objects. Pal [24] explained that both these TCP and TRP are interchangeable in the presence of consciousness to complete the 'process of observation'. Pal [24] explained further that these TCP and TRP are interchangeable in the presence of consciousness to carry and retain a specific 'thought' and also for its communication from one person to another. These TCP and TRP function like wavicle: wave-particle duality. Pal et al $[1,3]$ have shown that TCP or TRP may be mathematically allotted a mass $\left(\mathrm{m}_{\mathrm{T}}\right)$ equivalent to $4.831 \times 10^{-37} \mathrm{~g}$ to $5.5 \times 10^{-37} \mathrm{~g}$.

When $\mathrm{m}_{\mathrm{T}}=$ quantized mass of the TCP $=5.5 \times 10^{-37} \mathrm{~g}$, then

in the case of present universe of $10^{57} \mathrm{~g}$, the calculated number of TCP (in the inherent presence of TRP) = $10^{57} / 5.5 \times 10^{-37} \cong 1.81818 X 10^{93} \mathrm{TCP}$ (in the inherent presence of TRP).

It is to be noted that the universe is ultimately made up of $10^{93} \mathrm{TCP}$ (in the inherent presence of TRP), of course, in the presence of Thought force (TF). This thought force (TF) is an expression of the universal consciousness that, in turn, exists along with the universe.

The quantized energy $\left(\varepsilon_{T}\right)$ of the TCP represents universal consciousness.

$\mathrm{h}=$ Reduced Planck constant $=$

$h / 2 \pi=6.63 \times 10^{-27}$ erg. sec $/ 2 \times 3.142=$

$1.055 \times 10^{-27} \mathrm{erg}$.sec
The universe with $10^{93}$ TCP (in the inherent presence of TRP) gives rise to a value of $\mathrm{h}=1.055 \times 10^{-27} \mathrm{erg}$. sec

1 TCP or TRP gives rise to a value of $\mathrm{h}=$ $1.055 \times 10^{-27} \mathrm{erg}$. sec $/ 10^{93} \cong 10^{-120} \mathrm{erg}$. sec $\cong 10^{-120} \mathrm{in}$ $\mathrm{h} \cong \Lambda_{\text {(cosmological constant). }}$

Thus $\mathrm{b}$ value of a TCP (in the inherent presence of TRP) in the case of the universe having $1.81818 X 10^{93} \mathrm{TCP}$ (in the inherent presence of TRP) $\cong 10^{-120} \mathrm{erg} . \mathrm{sec} \cong 10^{-120}$ in $\mathrm{h} \cong \Lambda$ (cosmological constant).

The functional existence of cosmological constant $(\Lambda)$ signifies the existence of TCP (in the inherent presence of TRP).

Pal [27] expressed: Using the upper limit of the cosmological constant, the vacuum energy in a cubic centimeter of free space has been estimated to be $10^{-15}$ Joules as indicated by Carroll [8] $\cong 10^{-16} \mathrm{erg} \cong \mathcal{E}_{T}=$ quantized energy of the TCP. This reference Carroll [8] will be found in the paper published by Pal [27].

(It is to be noted that $\left.1 e V \cong 1.6021 \times 10^{-12} \mathrm{erg} \cong 1.6021 \times 10^{-19} \mathrm{~J}\right)$.

This expression also signifies the existence of TCP (in the inherent presence of TRP) to show the existence and function of cosmological constant $(\Lambda)$.

\subsubsection{Pal [27] Expressed}

In 1922, Friedmann of St Petersburg found that Einstein's equations do not permit a static Universe: the universe must be in motion, either expanding or contracting This contradicted Einstein's belief that the Universe must be eternal in time as well as uniform in space. To remedy this, Einstein introduced the so-called 'cosmological constant' which was physically equivalent to the assumption of a "universal repulsion" between material bodies. In contrast to other physical forces, which always decrease with distance, cosmological repulsion was assumed to be very weak over short distances but important at intergalactic distances. Gamow [9] explained the mathematical expression for this repulsive force acting on a particle of the mass $m$ as

$$
F=-\left(\frac{1}{3}\right) c^{2} \Lambda m r
$$

$$
\text { where } \quad \mathrm{c}=\text { velocity of light }
$$

$\Lambda=$ Einstein's cosmological constant

$\mathrm{r}=$ distance to the particle.

NOTE: This reference Gamow [9] will be found in the paper published by Pal [27].

Dutta [10] indicated that for the Einstein-de Sitter universe the radius $(\mathrm{R})$ of the universe is given as 


$$
R=\frac{2}{(4 \Lambda / 3)^{1 / 2}}
$$

NOTE: This reference Dutta [10] will be found in the paper published by Pal [27].

When $R=1.482 \times 10^{29} \mathrm{~cm}$, then it is found that

$\Lambda=$ Einstein's cosmological constant = $3 / R^{2}=1.3659186 \times 10^{-58} \mathrm{~cm}^{-2}$

Again, when $\Lambda=1.3659186 \times 10^{-58} \mathrm{~cm}^{-2}$,

$\mathrm{m}=\mathrm{m}_{\mathrm{T}}=$ quantized mass of the $\mathrm{TCP}=$ $5.5 \times 10^{-37} \mathrm{~g}$ and

$\mathrm{r}=\mathrm{R}=$ radius of the universe $=1.482 \times 10^{29} \mathrm{~cm}$, then it is found from the Equation (7) that

$$
F=-3.34 \times 10^{-45} \text { dyne }
$$

It is interesting to note that when $\varepsilon_{T}=4.95 \times 10^{-16} \mathrm{erg}$ and $D_{i}=R$ (radius of the present universe) $=1.482 \times 10^{29} \mathrm{~cm}$ in the macrocosm, the Equation (6) yields

$$
\mathrm{T}_{\mathrm{F}}(\text { macro })=\mathcal{E}_{T} / D_{i}=3.34 \times 10^{-45} \text { dyne. }
$$

Thus the value of $F$ in the Equation (9) is numerically exactly equal to that of $\mathrm{T}_{\mathrm{F}}$ (macro) as shown above.

Further,

$\mathrm{T}_{\mathrm{F}}($ macro $)=$

$$
\begin{aligned}
& \mathcal{E}_{T} / R=\varepsilon_{T} H_{0} / c= \\
& 3.34 \times 10^{-45} \text { dyne }
\end{aligned}
$$

Thus, the universal repulsion $(\mathrm{F})$ is equivalent to $\mathrm{T}_{\mathrm{F}}$ (macro) with a negative sign. If this $\mathrm{T}_{\mathrm{F}}$ (macro) functions as a repulsive force, then it may be found to be responsible for causing the expansion of universe.

As per Gamow [9], the relationship between recession velocity and distance is given by Hubble's law as

[Recession velocity] $=$ constant $\mathrm{x} \mathrm{D}$

$$
\text { i.e. } R V=\text { Ho } x D
$$

where $\quad \mathrm{RV}=$ recession velocity

$\mathrm{Ho}=$ Hubble's parameter $=\mathrm{c} / \mathrm{R}$

$\mathrm{D}=$ distance.

Putting $\quad H_{0}=3.34 \times 10^{-45}\left(c / \varepsilon_{T}\right)$ dyne from the Equation (10) in the Equation (11) one can get,

$$
\begin{aligned}
& R V=3.34 \times 10^{-45}\left(c / \varepsilon_{T}\right) \text { dynex } D= \\
& \left(c / \varepsilon_{T}\right) x T_{F}(\text { macro }) x D
\end{aligned}
$$

It is thus found that $\mathrm{T}_{\mathrm{F}}$ (macro) is related with the recession velocity (RV).

\subsubsection{Present Discussion}

It is shown that the universal repulsion $(\mathrm{F})$ is equivalent to $\mathrm{T}_{\mathrm{F}}$ (macro) with a negative sign. If this $\mathrm{T}_{\mathrm{F}}$ (macro) functions as a repulsive force, then it may be found to be responsible for causing the expansion of universe.

It is found from the Equation (12) that $T_{F}$ (macro) is related with the recession velocity (RV). This $R V$ is maintained by $\mathrm{T}_{\mathrm{F}}$ (macro) as well as $\mathcal{E}_{T}$, the quantized energy of TCP and c (velocity of light). As c, $\mathcal{E}_{T}$ and $\mathrm{T}_{\mathrm{F}}$ (macro) exist in this universe through the existence of the quantum mechanical activities of these TCP and TRP, so the universe is liable to expand incessantly through the maintenance of recession velocity in the inherent functional presence of TCP, TRP and $\mathrm{T}_{\mathrm{F}}$ (macro).

It has already been indicated that the functional existence of cosmological constant $(\Lambda)$ signifies the existence of TCP in the inherent presence of TRP.

Thus, the consciousness model involving TCP, TRP and $\mathrm{T}_{\mathrm{F}}$ (macro) can provide guidelines to explain the cause for the expansion of universe through its correlation with the cosmological constant $(\Lambda)$.

\subsubsection{Pal [28] Showed that the Acceleration of $T_{F}$ (macro)} is Found to be $6.173 \times 10^{-9} \mathrm{~cm} / \mathrm{sec}^{2}$.

Pal [28] showed that in the case of TF (macro) $=$ $\varepsilon_{T} / D_{i}=2.958 \times 10^{-45}$ dyne [within $D_{i}=R$ (radius of the present universe) $=1.482 \times 10^{29} \mathrm{~cm}$ in the macrocosm], using $\mathrm{m}=\mathrm{m}_{\mathrm{T}}=4.871 \times 10^{-37} \mathrm{~g}$, we get a $($ acceleration $)=\mathrm{F} / \mathrm{m}_{\mathrm{T}}=2.958 \mathrm{x} 10^{-45} / 4.871 \times 10^{-37}=$ $0.6173 \times 10^{-8} \mathrm{~cm} / \mathrm{sec}^{2}=6.173 \times 10^{-9} \mathrm{~cm} / \mathrm{sec}^{2}$.

Thus, in the case of TF (macro), a (acceleration) = $6.173 \times 10^{-9} \mathrm{~cm} / \mathrm{sec}^{2}$.

Pal [27] showed that the universal repulsion $(\mathrm{F})$ is equivalent to $T_{F}$ (macro) with a negative sign. If this $T_{F}$ (macro) functions as a repulsive force, then it may be found to be responsible for causing the expansion of universe. Here it is found that in the case of $\mathrm{T}_{\mathrm{F}}$ (macro), a $($ acceleration $)=6.173 \times 10^{-9} \mathrm{~cm} / \mathrm{sec}^{2}$.

\section{Discussion and Conclusion}

\subsection{Discussion}

A consciousness model involving TCP, TRP and thought force $\left(T_{F}\right)$ is developed in a purpose to form a possible gross bridge between mind and matter, present physics and cognitive science, psychology and natural sciences, classical physics and quantum physics.

Covering many enigmatic specific critical aspects of modern science I have published many research articles in Journals like American Journal of Modern Physics, American Journal of Physics and Applications and American Journal of Physical Chemistry: All these Journals are open access Journals of Science Publishing Group.

These articles, in my view, can provide plausible guidelines to tackle many present day scientific enigmas through this consciousness model.

There are varieties of published research articles in different journals. The titles of published research articles are as follows: 
1. Dhananjay Pal, Interpretation of the evolution of universe through the consciousness model, American Journal of Modern Physics. Vol. 2, No. 6, 2013, pp. 303313. doi: 10.11648/j.ajmp.20130206.16

2. Dhananjay Pal, Existence of Thought Force and Its Characteristics, American Journal of Physical Chemistry. Vol. 2, No. 5, 2013, pp. 94-104. doi: 10.11648/j.ajpc.20130205.13

3. Dhananjay Pal, Sense of Time and Interpretation of the Evolution of Time, American Journal of Modern Physics. Vol. 2, No. 6, 2013, pp. 314-321. doi: 10.11648/j.ajmp.20130206.17

4. Dhananjay Pal, Possible Bridging the Classical Physics and Quantum Physics through Consciousness,

American Journal of Modern Physics. Vol. 2, No. 6, 2013, pp. 322-329. doi: $\quad$ 10.11648/j.ajmp.20130206.18

5. Dhananjay Pal, Developing an Equation to Compute the Wavelength $(\lambda \mathrm{g})$ and Quantized Energy $(\mathrm{Eg})$ of Elusive Graviton, American Journal of Modern Physics. Vol. 2, No. 6, 2013, pp. 330-333. doi: 10.11648/j.ajmp.20130206.19

6. Dhananjay Pal, Interpretation of Information Processing and Mode of Perception Through the Consciousness Model Involving Though-Carrying Particle (TCP), Thought Retaining Particle (TRP) and Thought Force (TF) in vitro and Thought Force (TF) in vivo, American Journal of Modern Physics. Vol. 2, No. 6, 2013, pp. 341-349. doi: 10.11648/j.ajmp.20130206.21

7. Dhananjay Pal, Some Specific Plausible Theoretical Aspects to Justify and Support the Existence of ThoughtCarrying Particle, Thought Retaining Particle and Thought Force, American Journal of Physical Chemistry. Vol. 2, No. 5, 2013, pp. 80-93. doi: 10.11648/j.ajpc.20130205.12

8. Dhananjay Pal, Interpretation of Quantum Measurement as Well as Cognitive Problem Through the Consciousness Model, American Journal of Physics and Applications. Vol. 1, No. 2, 2013, pp. 38-44. doi: 10.11648/j.ajpa.20130102.12

9. Dhananjay Pal, Role of Thought Force (TF) of Consciousness Model Along with Gravitational Force (GF) to Explain the Relevant Conditions for the Formation of A Black Hole, American Journal of Physics and Applications. Vol. 1, No. 3, 2013, pp. 50-54. doi: 10.11648/j.ajpa.20130103.11

10. Dhananjay Pal, Interpretation of the Cause for the Expansion of Universe Having Its Correlation with the Cosmological Constant $(\Lambda)$ through the Consciousness Model, American Journal of Physics and Applications. Vol. 1, No. 2, 2013, pp. 45-49. doi: 10.11648/j.ajpa.20130102.13

11. Dhananjay Pal, Application of the Consciousness
Model Involving Thought-Carrying Particle (TCP), Thought Retaining Particle (TRP) and Thought Force (TF) to Explain the Cause of Radioactivity, American Journal of Physics and Applications. Vol. 1, No. 3, 2013, pp. 55-58. doi: $10.11648 /$ j.ajpa.20130103.12

12. Dhananjay Pal, Explanation of Mental Processes Involving Consciousness through the Consciousness Model Incorporating Quantum Mechanical Activities of ThoughCarrying Particle (TCP) and Thought Retaining Particle (TRP) in the Presence of Thought Force (TF) in vitro and Thought Force (TF) in vivo, American Journal of Physics and Applications. Vol. 1, No. 3, 2013, pp. 59-66. doi: 10.11648/j.ajpa.20130103.13

13. Dhananjay Pal, Relation of Space-Time Continuum with Energy in the Presence of Consciousness and SuperConsciousness, American Journal of Physics and Applications. Vol. 1, No. 3, 2013, pp. 67-79. doi: 10.11648/j.ajpa.20130103.14

14. Dhananjay Pal, Interpretation of the Non-Locality Problem in Quantum Mechanics through the Consciousness Model, American Journal of Physics and Applications. Vol. 2, No. 1, 2014, pp. 1-5. doi: 10.11648/j.ajpa.20140201.11

15. Dhananjay Pal, Interpretation of the Evolution of Life and Consciousness through the Consciousness Model, American Journal of Physics and Applications. Vol. 2, No. 1, 2014, pp. 6-18. doi: 10.11648/j.ajpa.20140201.12

16. Dhananjay Pal, Interpretation of Mind and Consciousness and Possible Interrelationship among Consciousness, Mind, Brain and Matter through the Consciousness Model, American Journal of Physics and Applications. Vol. 2, No. 1, 2014, pp. 19-30. doi: 10.11648/j.ajpa.20140201.13

17. Dhananjay Pal, Possible Bridge between the Mind and Matter: The Physical World is nothing but a Projection of the Mind Consisting of TCP and TRP that, in Turn, are the Ultimate Constituents of any Matter also, American Journal of Physical Chemistry. Vol. 2, No. 5, 2013, pp. 105-116. doi: 10.11648/j.ajpc.20130205.14

18. Dhananjay Pal, Interpretation of the Paranormal Power Like Extra Sensory Perception (ESP) and Psychokinesis (PK) through the Consciousness Model, American Journal of Physics and Applications. Vol. 1, No. 3, 2013, pp. 80-90. doi: 10.11648/j.ajpa.20130103.15

19. Dhananjay Pal, The Relationship between consciousness and Vital Living Force through the Consciousness Model, American Journal of Modern Physics. Vol. 3, No. 1, 2014, pp. 1-7. doi: 10.11648/j.ajmp.20140301.11

20. Dhananjay Pal, The Observed Change in the Value of 'Fine Structure Constant' can be Interpreted through the 
Consciousness Model, American Journal of Modern Physics. Vol. 3, No. 1, 2014, pp. 8-15. doi: 10.11648/j.ajmp.20140301.12

21. Dhananjay Pal, Theoretical Characteristics of Though-Carrying Particle (TCP) and Thought Retaining Particle (TRP) Are Necessary for Providing Plausible Possible Answers to the Typical Specific Questions Raised by Different Learned Reviewers, American Journal of Modern Physics. Vol. 2, No. 6, 2013, pp. 362-374. doi: 10.11648/j.ajmp.20130206.24

22. Dhananjay Pal, The Mental Impression and the Doctrine of Reincarnation Leading to the Characteristic Hereditary Transmissions through the Consciousness Model, American Journal of Modern Physics. Vol. 2, No. 6, 2013, pp. 392-401. doi: 10.11648/j.ajmp.20130206.27

23. Dhananjay Pal, The Healing Power of Mind and Theoretical Approach to Develop a So-Called Panacea through the Consciousness Model, American Journal of Modern Physics. Vol. 2, No. 6, 2013, pp. 402-416. doi: 10.11648/j.ajmp.20130206.28

\subsection{Conclusion}

Formation of a possible gross bridge between mind and matter, present physics and cognitive science, psychology and natural sciences, classical physics and quantum physics through the consciousness model has been expressed thoroughly by mentioning many published papers dealing with these aspects.

The existence of TCP, TRP and thought force $\left(\mathrm{T}_{\mathrm{F}}\right)$ in vitro and thought force $\left(\mathrm{T}_{\mathrm{F}}\right)$ in vivo may provide plausible guidelines to lucidly explain the evolution of the universe with fundamental laws of natural science like physics, chemistry and biology (with consciousness).

It appears that the standard model of physics is to be correctly tuned by ascertaining the constituents of quarks and leptons in the presence of unified field in order to form a possible gross bridge between mind and matter, present physics and cognitive science, psychology and natural sciences, classical physics and quantum physics.

\section{References}

[1] D. Pal and A.U. De, Physics of consciousness and its model may provide guidelines to solve Many scientific problems. Neuroquantology 1: 17-28(2004)

[2] D. Pal and A.U. De, Consciousness model: Significance of thought-carrying particles and thought-retaining particles in quantum measurement as well as cognitive problem. Neuroquantology 2: 115-116 (2005)

[3] D. Pal and A.U. De, The cosmic microwave background radiation temperature signifying the existence of the thought-carrying particle, thought retaining particle and thought force. NeuroQuantology 10: Issue3; 428-442 (September 2012)

[4] M. Bhaumik, Code Name GOD. (Penguin Books India Pvt.
Ltd., 11 Community Centre, Panchsheel Park, New Delhi 110017 , India) pp. 132-133; 161-162; 167; 171; 177; 183 and 184. 185-186; 89-190; 198 (2006)

[5] Dhananjay Pal, Existence of Thought Force and Its Characteristics, American Journal of Physical Chemistry. Vol. 2, No. 5, 2013, pp. 94-104. doi: 10.11648/j.ajpc.20130205.13

[6] V. F. Weisskopf, The Origin of the Universe, The World of Physics, (Simon and Schuster, 1230 Avenue of Americas, New York 10020) 3, pp. 10 and 314 (1987)

[7] D. Pal, Existence of universal consciousness and its characteristics. Accepted for publication in 2014 International Conference on Advanced Education and Management (ICAEM2014) Beijing, China (2013)

[8] I. Prigogine and G. Nicolis, Self-Organization in NonEquilibrium Systems. (Wiley 1977) ISBN 0471024015.

[9] R. Nelson, Director, Global Consciousness Project, Princeton, New Jersey. (2009) rdnelson@princeton.edu

[10] D. Radin, The Conscious Universe: The Scientific Truth of Psychic Phenomena. (Harper Edge) (1997) ISBN 0-06251502-0.

[11] R. Penrose, Shadows of the Mind: A Search for the Missing Science of Consciousness. (Oxford University Press) (1994)

[12] W. Van De Bogart, Earth portals: Exploring New Metaphors of Consciousness 1993 willard@earthportals.com

[13] S. Vivekananda, The Cosmos (the Microcosm). The complete works of Swami Vivekananda. (Advaita Ashrama, India) 2: pp-212 (1989)

[14] S. Parnia, Do reports of consciousness during cardiac arrest hold the key to discovering the nature of consciousness? Medical Hypotheses 69(4): 933-937 (2007)

[15] P van. Lommel, R. Wees, V. Meyers and I. Elfferich, NearDeath Experience in Survivors of Cardiac Arrest: A prospective Study in the Netherlands. The Lancet (9298): 2039-45 (2001)

[16] P. Fenwick, S. Parnia, D.G. Waller, and R. Yeates, A qualitative and quantitative study of the incidence, features and aetiology of near-death experiences in cardiac arrest survivors. Resuscitation 48: 149-156 (2001)

[17] M. Beauregard and D. O'Leary, The spiritual brain: A neuroscientist's case for the existence of the soul. (Harper Collins Publishers) (2008)

[18] M. Beauregard and V. Paquette, Neural correlates of a mystical experience in Carmelite nuns. Neuroscience Letters 405, 186-190 (2006)

[19] L. Cosmides and J. Tooby J. From evolution to behavior: Evolutionary psychology as the missing link. (In J. Dupre (Ed.), The latest on the best: Essays on evolution and optimality, Cambridge, MA, The MIT Press. (1987)

[20] L. Cosmides, J. Tooby, J. Barkow, The adapted mind: Evolutionary psychology and the generation of culture. (New York: Oxford University Press. 1992)

[21] L. Cosmides and J. Tooby, Beyond intuition and instinct blindness: Towards an evolutionary rigorous cognitive science. Cognition; 50 (1-3): 41-77 (1994) 
124 Dhananjay Pal: The Developed Consciousness Model Providing Plausible Guidelines to Form a Possible Gross Bridge between Mind and Matter, Present Physics and Cognitive Science, Psychology and Natural Sciences, Classical Physics and Quantum Physics

[22] BD. Josephson. Biological Utilization of Quantum Nonlocality. Foundations in Physics, Vol. 21; 197-207. (1991)

[23] Dhananjay Pal, Possible Bridge between the Mind and Matter: The Physical World is nothing but a Projection of the Mind Consisting of TCP and TRP that, in Turn, are the Ultimate Constituents of any Matter also, American Journal of Physical Chemistry. Vol. 2, No. 5, 2013, pp. 105-116. doi: 10.11648/j.ajpc.20130205.14

[24] Dhananjay Pal, Interpretation of Quantum Measurement as Well as Cognitive Problem Through the Consciousness Model, American Journal of Physics and Applications. Vol. 1, No. 2, 2013, pp. 38-44. doi: 10.11648/j.ajpa.20130102.12

[25] Dhananjay Pal, Interpretation of the Paranormal Power Like Extra Sensory Perception (ESP) and Psychokinesis (PK) through the Consciousness Model, American Journal of Physics and Applications. Vol. 1,
No.
3 ,
2013,
pp.
80-90.
doi:

\subsection{8/j.ajpa.20130103.15}

[26] Dhananjay Pal, Possible Bridging the Classical Physics and Quantum Physics through Consciousness, American Journal of Modern Physics. Vol. 2, No. 6, 2013, pp. 322-329. doi: 10.11648/j.ajmp.20130206.18

[27] Dhananjay Pal, Interpretation of the Cause for the Expansion of Universe Having Its Correlation with the Cosmological Constant $(\Lambda)$ through the Consciousness Model, American Journal of Physics and Applications. Vol. 1, No. 2, 2013, pp. 45-49. doi: 10.11648/j.ajpa.20130102.13

[28] Dhananjay Pal, Theoretical Characteristics of ThoughCarrying Particle (TCP) and Thought Retaining Particle (TRP) Are Necessary for Providing Plausible Possible Answers to the Typical Specific Questions Raised by Different Learned Reviewers, American Journal of Modern Physics. Vol. 2, No. 6, 2013, pp. 362-374. doi: 10.11648/j.ajmp.20130206.24 